\title{
A Robust DOA Estimation Based on Sigmoid Transform in Alpha Stable Noise Environment
}

\author{
$\mathrm{Li} \mathrm{Li}^{1, *}$, Mingyan $\mathrm{He}^{1}$ \\ ${ }^{1}$ Information Engineering College, Dalian University, 116622, Dalian, China
}

\begin{abstract}
To suppress alpha-stable noise and co-channel interference, this paper defines a novel cyclic correlation function, and proposes a new MUSIC algorithm based on sigmoid cyclic correlation function. Furthermore, the proposed algorithm (SCC-MUSIC) is applied to estimate direction of arrival (DOA) in alpha stable distribution noise. Simulation results demonstrate that the proposed SCC-MUSIC can get a better performance than several existing algorithms especially in the highly impulsive noise environments.
\end{abstract}

\section{Introduction}

Source localization by direction of arrival (DOA) has been received considerable attention in radar, sonar and wireless communication over the past years ${ }^{[1]}$. The MUSIC algorithm ${ }^{[2]}$, one of the most well-known subspace methodology, has attracted much attention and is an asymptotically unbiased DOA estimator based on the Gaussian noise assumption ${ }^{[3,4]}$. However, in some scenarios, noises exhibit an impulsive nature caused mainly by sudden bursts or sharp spikes ${ }^{[5-7]}$, and it is inappropriate to model the noise as Gaussian distributed.

To overcome the Alpha stable distribution noise, many DOA estimation algorithms based on the fractional lower-order statistics (FLOS) were proposed [8-10]. However, the algorithms have obtained certain estimation preformation, but have some limitations: (1) the characteristic exponent of noise must be estimated to ensure $1 \leq p<\alpha$ or $0<p<\alpha / 2$, where $p$ is the order of moments and $\alpha$ is the characteristic exponent of the impulsive noise. (2) these methods can not accurately estimate parameter if there is not the priori knowledge of characteristic exponent, furthermore, algorithms preformation degrade seriously even invalid while fractional lower-order moment value is not appropriate. To handle this problem, [11] proposes an algorithm based M-estimate like correlation algorithm to estimate DOA. Zhang and Qiu ${ }^{[12,13]}$ propose the algorithm of DOA estimation based CRCO-MUSIC. However, performance of these algorithms will degrade significantly when the underlying noise is highly impulsive, specially $\alpha<1$.

Most man-made signals encountered in communication, telemetry, radar and sonar systems, some parameters do vary periodically with time. There can be much to gain in terms of improvements in performance of these processors by recognizing and exploiting the very special property shared by many man-made signals named as cyclostationarity ${ }^{[14-16]}$. The cyclostationary property can be exploited to cancel interference and background noise. There are many kinds of noise and interference in wireless communication, telemetry, radar and sonar systems, and the received signal may be submerged. To suppress impulse noise and co-channel interference, this paper defines a novel cyclic correlation function and propose a new algorithm based sigmoid cyclic correlation to estimate DOA. The proposed method is simple and effective. Simulations verified the superiority of the proposed estimation method over existing methods in the presence of both alpha-stable distribution noise and cochannel interference.

\section{Signal model and sigmoid transform}

\subsection{Signal model}

Consider $L$ statistically independent narrowband planewave signals impinge on a uniformly linear array (ULA) of $M$ identical omni-directional sensors. The sensors and signals are assumed to be co-planar since our discussion is confined to azimuth-only systems. We assume only $K_{a}(<\mathrm{L})$ signals of interest (SOIs) exhibit a cyclic property at the cycle frequency $\varepsilon$. The other $L-K_{a}$ signals either have different cycle frequencies or do not exhibit cyclostationarity. The vector form of the array output can be shown as [11-12]

$$
\boldsymbol{X}(t)=\boldsymbol{A}(\theta) \boldsymbol{S}(t)+\boldsymbol{N}(t)
$$

where $\boldsymbol{X}(t)=\left[x_{1}(t), \ldots, x_{M}(t)\right]^{\mathrm{T}}$ is the $M \times 1$ received signal vector, $S(t)=\left[s_{1}(t), s_{2}(t), \cdots s_{K_{a}}(t)\right]^{\mathrm{T}} \quad$ denotes transmitted signal vector with the cycle frequency $\varepsilon$, and $A(\theta)=\left[a\left(\theta_{1}\right), a\left(\theta_{2}\right), \ldots, a\left(\theta_{k_{a}}\right)\right]$ contains interfering sources and alpha stable distribution noise. Furthermore, $a\left(\theta_{k}\right)=\left[1, e^{j 2 \pi \sin \theta_{k} d / \lambda}, \ldots, e^{j 2 \pi \sin \theta_{k}(M-1) d / \lambda}\right]$ 
$\mathbf{N}(t)=\left[n_{1}(t), \cdots, n_{M}(t)\right]^{\mathrm{T}}$ is the $M \times 1$ noise vector, in which $n_{m}(t)$ is a sequence of i.i.d isotropic complex $S \alpha S$ random variable with $1<\alpha \leq 2 ; S \alpha S$ can be conveniently described by its characteristic function, as follows

$$
\phi(\xi)=\mathrm{e}^{\mathrm{j} \mu \xi-\gamma|\xi|^{\alpha}}
$$

where $\alpha \in(0,2]$ is the characteristic exponent, which can measure the heaviness of the distribution tail. The smaller $\alpha$ is, the heavier its tail is. If $\alpha=2$, Eq.(2) could also describe the Gaussian distribution. The random variables of $S \alpha S$ have no $p$-th-order moments when $p>\alpha . \gamma$ is the dispersion parameter. $\mu$ is the location parameter; it depicts the mean value when $1<\alpha \leq 2$ or the median value when $0<\alpha<1$.

\subsection{Sigmoid function}

The Sigmoid function is widely accepted as a common nonlinear transformation ${ }^{[17]}$. Its definition is shown in Eq.(3).

$$
w_{x}(t)=\frac{2}{1+\exp [-x(t)]}-1
$$

where $\lambda$ is the inclined coefficient to adjust $x(t)$ at different scales.

Three results are obtained from the analysis of the Sigmoid function transform ${ }^{\text {[17]: }}$

Results 1: If $x(t)$ is a $S \alpha S$ process with $\beta=0$, and $a=0$, then $w_{x}(t)$ is symmetric distribution with zero mean in its probability density function.

Results 2: If $x(t)$ is a $S \alpha S$ process with $\gamma>0$, and $a=0$, then we have $\|w(t)\|_{\alpha}>0$, and the mean value of $w_{x}(t)$ is zero.

Results 3: If $x(t)$ is a $S \alpha S$ process with $a=0$, then $w_{x}(t)$ has the finite second order moment with zero mean (referred to as second order moment process).

A novel cyclic correlation $R_{x, \text { Sigmoid }}^{\varepsilon}(\tau)$, referred to as the Sigmoid based cyclic correlation (SCC), is defined in Eq. (4)

$$
R_{x, \text { Sigmoid }}^{\varepsilon}(\tau)=\left\langle w_{x}(t+\tau / 2) w_{x}^{*}(t-\tau / 2) e^{-j 2 \pi \varepsilon t}\right\rangle_{t}
$$

where $\langle\cdot\rangle_{t}$ represents time average.

\section{SCC-MUSIC algorithm based DOA estimation}

According Eq. (3) and Eq. (4), we can obtain the sigmoid cyclic correlation function $\mathbf{R}_{\mathbf{X} \text {,Sigmoid }}^{\varepsilon}(\tau)$ of the received signal vector $\mathbf{X}(t)$,

$$
\begin{aligned}
\mathbf{R}_{\mathbf{X}, \text { Sigmoid }}^{\varepsilon}(\tau) & =\left\langle w_{\mathbf{X}}(t+\tau / 2) w_{\mathbf{X}}^{*}(t-\tau / 2) e^{-j 2 \pi \varepsilon t}\right\rangle_{t} \\
& =\mathbf{A R}_{\mathbf{S}, \text { Sigmoid }}^{\varepsilon}(\tau) \mathbf{A}^{\mathrm{H}}+\mathbf{A R}_{\mathbf{S N}^{*}, \text { Sigmoid }}^{\varepsilon}(\tau) \\
& +\mathbf{R}_{\mathbf{S}, \text { Sigmoid }}^{\varepsilon}(\tau) \mathbf{A}^{\mathrm{H}}+\mathbf{R}_{\mathbf{N}, \text { Sigmoid }}^{\varepsilon}(\tau)
\end{aligned}
$$

where

$$
\mathbf{R}_{\mathbf{S}, \text { Sigmoid }}^{\varepsilon}(\tau)=\left\langle w_{\mathbf{S}}(t+\tau / 2) w_{\mathbf{S}}^{*}(t-\tau / 2) e^{-j 2 \pi \varepsilon t}\right\rangle_{t}
$$

denotes the sigmoid cyclic autocorrelation of transmitted signal vector $\mathbf{S}(t)$. We find that the correlation matrix estimation used in subspace algorithms is replaced by the sigmoid cyclic correlation matrix estimation. Since the sigmoid transformation can suppress $\alpha$-stable noise, and the cyclostationarity of the signal can select the SOIs, the number of sources presented in the sigmoid cyclic correlation matrix is reduced, thus the detection capability and resolution performance can be significantly improved.

The noise $n(t)$ is statistically independent with zeromean values, and exhibit no cyclostationarity with cyclic frequency $\varepsilon$, and the transmitted signal $s(t)$ is cyclostationarity independent of noise $n(t)$, so Eq.(5) can be rewritten as

$$
\mathbf{R}_{\mathbf{X}, \text { Sigmoid }}^{\varepsilon}(\tau)=\mathbf{A} \mathbf{R}_{\mathbf{S}, \text { Sigmoid }}^{\varepsilon}(\tau) \mathbf{A}^{\mathrm{H}}
$$

The singular value decomposition (SVD) of the sigmoid cyclic correlation matrix $\mathbf{R}_{\mathbf{x} \text {, Sigmoid }}^{\varepsilon}(\tau)\left(K_{a}<M\right)$,

$$
\mathbf{R}_{\mathbf{X}, \text { Sigmoid }}^{\varepsilon}(\tau)=\mathbf{U S V}^{\mathrm{H}}
$$

For the transmitted signal $s(t)$ is cyclostationarity independent of noise $n(t), \operatorname{rank}\left(\mathbf{R}_{\mathbf{x}, \text { Sigmoid }}^{\varepsilon}(\tau)\right)$ $=\operatorname{rank}\left(\mathbf{R}_{\mathrm{S} \text {,Sigmoid }}^{\varepsilon}(\tau)\right)=K_{a}$, we can get a left singular subspace by the $K_{a}$ left singular vectors associated with the $K_{a}$ nonzero singular values. Then the $M-K_{a}$ singular vectors associated with the zero singular values (in practice, there is usually no zero singular value but instead s small singular value) span the noise subspace.

The Eq.(6) is denoted by

$$
\begin{aligned}
\mathbf{R}_{X, \text { Sigmoid }}^{\varepsilon}(\tau) & =\left[\begin{array}{ll}
\mathbf{U}_{\mathbf{s}} & \mathbf{U}_{\mathbf{N}}
\end{array}\right]\left[\begin{array}{cc}
\sum_{\mathbf{s}} & 0 \\
0 & \sum_{\mathbf{N}}
\end{array}\right]\left[\begin{array}{ll}
\mathbf{V}_{\mathbf{S}} & \mathbf{V}_{\mathbf{N}}
\end{array}\right]^{H} \\
& =\mathbf{U}_{\mathbf{S}} \sum_{S} \mathbf{V}_{S}^{H}
\end{aligned}
$$

Where $\left[\begin{array}{ll}\mathbf{U}_{\mathbf{S}} & \mathbf{U}_{\mathbf{N}}\end{array}\right]$ and $\left[\begin{array}{ll}\mathbf{V}_{\mathbf{S}} & \mathbf{V}_{\mathbf{N}}\end{array}\right]$ are unitary, and the diagonal elements of diagonal matrix $\sum_{s}$ are positive. The column vectors of $\boldsymbol{U}_{S}$ and $\boldsymbol{U}_{N}$ are the eigen vectors spanning the signal subspace and noise subspace of $\mathbf{R}_{\mathbf{x} \text {,Sigmoid }}^{\varepsilon}(\tau)$, respectively, with the associated eigen values on the diagonals of $\sum_{\mathrm{s}}$ and $\sum_{\mathrm{N}}$. As the signal $S$ is independent of the noise $N$ and signal subspaces is orthogonal with noise subspaces, therefore, spatial spectrum of SCC-MUSIC can be got based on classical MUSIC algorithm, which can be expressed as

$$
P(\theta)=\frac{1}{\boldsymbol{a}^{\mathrm{H}}(\theta) \boldsymbol{U}_{N} \boldsymbol{U}_{N}^{\mathrm{H}} \boldsymbol{a}(\theta)}
$$

Searching spectral peak of $P(\theta)$, we can get the DOA estimator $\theta_{l}$.

\section{Simulation results}

In the simulations, we consider a ULA with $M=8$ elements. Suppose the carrier frequency of the narrow- 
band SOI with the DOA $\left(\theta_{1}=0^{\circ}\right.$ and $\theta_{2}=40^{\circ}$.) is $f_{c 1}=100 \mathrm{MHz}$, the other signal is considered as interference, whose carrier frequency is $f_{c 2}=150 \mathrm{MHz}$ with $\mathrm{DOA}=\left(10^{\circ}, 20^{\circ}\right)$. The percentage bandwidth (the ratio of the bandwidth to the carrier frequency) of both SOI and interference is all $1 \%$. The cyclic frequency of BPSK signal is $\varepsilon= \pm 2 f_{0}+\frac{k}{T_{C}}(k=0, \pm 1, \pm 2, \ldots)^{[15-16]}$, where $f_{0}$ is the carrier frequency, and the reciprocal of Tc is baud rate. In this simulation, we adopt $\varepsilon=2 f_{c 1}$. Since $\alpha$ stable distribution with $\alpha<2$ determines infinite variance, we describe the signal-to-noise condition of $S \alpha S$ using the generalized signal-noise-ratio (GSNR). The GSNR is defined by

$$
\operatorname{GSNR}=10 \lg \left(\sigma_{\mathrm{s}}^{2} / \gamma\right)
$$

where $\sigma_{\mathrm{s}}^{2}$ and $\gamma$ are the variance of the underlying signal and dispersion of the $S \alpha S$ noise, respectively.

The performance is evaluated by two statistical performance measures: the probability of resolution and root mean square error (RMSE) of DOA estimation. If the DOA estimation error for each source is less than $3^{\circ}$ simultaneously, we call it a successful estimation. The probability of resolution is the ratio of the successful runs to the total Monte Carlo runs and the RMSE of those successful runs is defined by

$$
\operatorname{RMSE}=\frac{1}{2}\left(\sqrt{\frac{1}{L} \sum_{l=1}^{K}\left[\hat{\theta}_{1}(l)-\theta_{1}\right]^{2}}+\sqrt{\frac{1}{L} \sum_{l=1}^{K}\left[\hat{\theta}_{2}(l)-\theta_{2}\right]^{2}}\right)
$$

where $\hat{\theta}_{1}$ and $\hat{\theta}_{2}$ are the successful estimation of $\theta_{1}$ and $\theta_{2}$, and $L$ is the total number of successful runs. Each experiment is executed by 500 Monte-Carlo runs.

In this section, some simulations have been carried out to compare the performance of different methods including conventional MUSIC ${ }^{[4]}$, FLOM-MUSIC [10], CRCO-MUSIC ${ }^{[15]}$, and the proposed SCC-MUSIC. The kernel size $\sigma$ is set as $\sigma=3$ for CRCO-MUSIC, and the parameter $p$ is set as $p=1.1$ for FLOM-MUSIC according to the simulation results and the conclusions.

\subsection{GSNR}

In this simulation, the generalized signal to noise ratio is set as $0 \leq G S N R \leq 10, \alpha=1.4$ and snapshot number is $N=100$. The influence of GSNR to the performance of four algorithms is shown in Fig.1. Although all the performances are improved with the increase of GSNR, the conventional MUSIC method is inferior to other four methods. For low GSNRs, both the CRCO-MUSIC and the SCC-MUSIC can get higher successful probability than other methods, however, the SCC-MUSIC can obtain more accurate DOA estimation results. For high GSNRs, the CRCO-MUSIC and MCC-MUSIC can obtain smaller RMSEs than other methods. Therefore, the SCC-MUSIC algorithm have better preformation especially when the GSNR values are low.

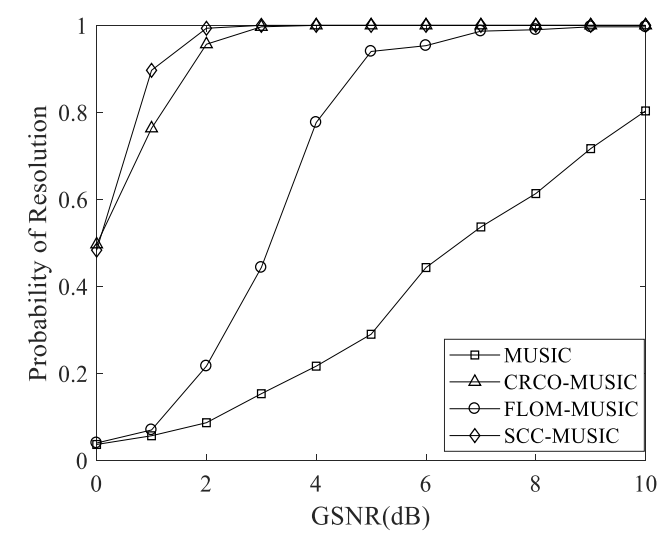

(a) Probability of resolution

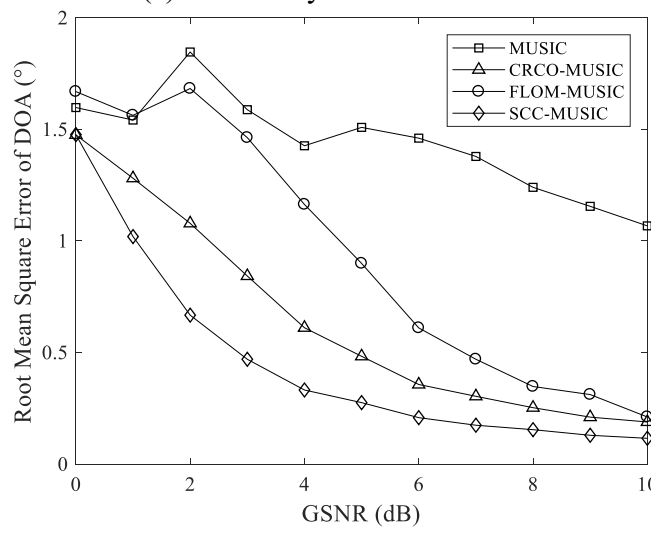

(b) Root mean square error

Fig.1. Probability of resolution and RMSE versus GSNR

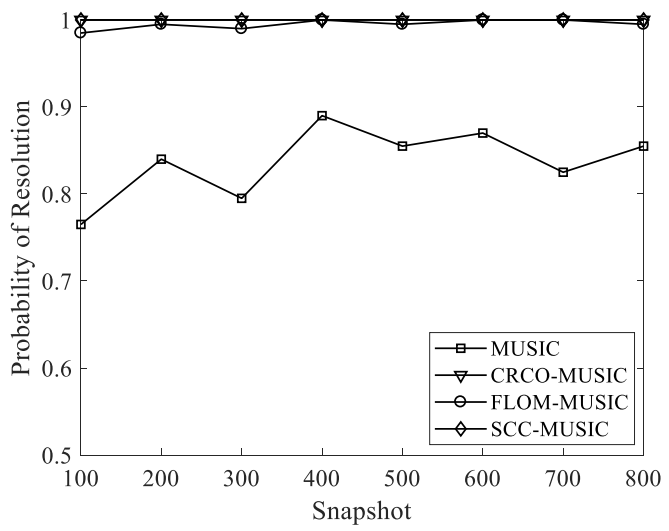

(a) Probability of resolution



(b) Root mean square error

Fig.2. Probability of resolution and RMSE versus Snapshot 


\subsection{Snapshot}

In this simulation, the generalized signal to noise ratio is set as GSNR $=10 \mathrm{~dB}$ and $\alpha=1.5$. The influence of the Snapshot to the performance of four algorithms are shown in Fig.2. From Fig.2, we can find the RMSE of other three methods decrease with the increase of snapshot number except conditional MUSIC method. So, we adapt $N=800$ for further simulations. Furthermore, these three methods have similar probability of resolution. However, the proposed method can obtain lower estimation RMSE.

\subsection{Characteristic exponent $\alpha$}

In this simulation, the generalized signal to noise ratio is set as $\mathrm{GSNR}=10 \mathrm{~dB}$ and $0.2 \leq \alpha \leq 2$. The influence of the Characteristic exponent to the performance of four algorithms are shown in Fig.3.

We can also see that the performance of the proposed method varies slightly with the variation of $\alpha$, and the proposed method can get a significantly higher successful probability and better estimation of DOA than those of other methods when $\alpha<1$. It means that the proposed method is more robust to highly impulsive noise. We also can find that the estimation accuracy of the proposed method is higher than other methods



(a) Probability of resolution



(b) Root mean square error

Fig.3. Probability of resolution and RMSE versus $\alpha$

\section{Conclusion}

Inspired by the theory of Sigmoid function, this paper defines the sigmoid cyclic-correlation function (SCC), and constructs cyclic correlation matrix based on the sigmoid transformation. The corresponding new estimation method (SCC-MUSIC) is proposed for DOA estimation under the $\alpha$-stable noise conditions. We compare performance of the proposed methods with those of conditional MUSIC, FLOM-MUSIC and CRCO-MUSIC algorithms under the condition of complex isotropic symmetric $\alpha$ stable noise model. The simulation results show that the proposed method can get better estimation results.

\section{Acknowledgements}

This work was partly supported by the National Natural Science Foundation of China under Grants 61401055 and 61671105, the Ph.D Programs Foundation of Liaoning Province of China 20170520421, and the China Scholarship Fund Program.

\section{References}

1. H. Krim, M.Viberg, IEEE Signal Proc. Mag. 13, 4 (1996).

2. R. O. Schmide. IEEE T. Antenn. Propag. 34, 3(1986).

3. B. Porat, B. Friedlander, IEEE Trans. Acoust., Speech, Signal Processing. 36, 4 (1988).

4. P. Stoica, N. Arye, IEEE Trans. Acoust., Speech, Signal Process. 37,5 (1989) .

5. M.D. Button, J.G. Gardiner, I.A. Glover, IEEE Trans. Veh. Technol. 51, 3 (2002).

6. Xu, Z., Yang, C., Tan, Z., and Sheng, Z. IEEE Commun. Lett. 21, 10 (2017).

7. S. Ghofrani, IET Signal Process. 8, 5 (2014).

8. M. Shao, C.L. Nikias, Proc. IEEE. 81, 7 (1993).

9. P. Tsakalides, C.L. Nikias, IEEE Trans. Signal Processing.44,7 (1996).

10. L. Tsung-Hsien, J.M. Mendel, IEEE Trans. Signal Process. 49, 8 (2001) .

11. J.F. Zhang, T.S. Qiu, S. Li, Acta Electronica Sinica, 43, 3 (2015).

12. J.F. Zhang, T.S. Qiu, A.M. Song, H. Tang, Signal Process. 104, 11(2014).

13. T.S. Qiu, J. F. Zhang, A. M. Song, Signals Process. 28, 4 (2012).

14. S.V. Schell, R.A. Calabretta, W.A. Gardner, B. G. Agee, IEEE Trans. Ac., Sp. Signal Process. 37, 4 (1989).

15. W.A. Gardner, IEEE Trans. Ac., Sp. Signal Process. 34, 5 (1986).

16. W.A. Gardner, W.A. Brown, C.K. Chen, IEEE Trans. Commun. 35, 6 (1987). 
17. T.S. Qiu, H. Wang, Y. Zhang. Methods Archive, 41,4 (2002). 